\title{
La filière lin oléagineux française : panorama et perspectives
}

\author{
Françoise LABALETTE ${ }^{1}$ \\ Nathalie LANDÉ ${ }^{2}$ \\ Dominique WAGNER ${ }^{2}$ \\ Martine ROUX-DUPARQUE ${ }^{3}$ \\ Eva SAILLET ${ }^{1}$ \\ 1 Onidol, \\ 12 avenue George-V, \\ 75008 Paris, \\ France \\ $<$ f.labalette@onidol.fr $>$ \\ ${ }^{2}$ Cetiom, \\ avenue Lucien-Brétignières, \\ 78850 Thiverval-Grignon, \\ France \\ ${ }^{3}$ Agro-transfert ressources et territoires, \\ 2, Chaussée de Brunehaut, \\ 80200 Estrées-Mons, \\ France
}

La production de lin oléagineux en France peine à se stabiliser et à satisfaire la demande d'opérateurs d'aval qui valorisent la graine riche en oméga 3 (acide alphalinolénique) dans des filières animales en vue de fournir des aliments (produits laitiers, œufs, jambon) enrichis naturellement en oméga 3. Et pourtant, les éléments de contexte sont favorables à un regain de cette culture : que ce soit un marché européen largement déficitaire en graines de lin oléagineux ou les évolutions environnementales demandées à l'agriculture. C'est pourquoi l'Organisation nationale interprofessionnelle des oléagineux (Onidol) et le Centre technique interprofessionnel des oléagineux métropolitains et du chanvre industriel (Cetiom) ont lancé en 2008 un diagnostic de la filière du lin français. Cette étude avait pour objectifs de conduire un état des lieux depuis la production jusqu'à I'utilisation du lin français et d'en déduire quelques perspectives pour le futur. Pour bénéficier

\begin{abstract}
Linseed oil crop production does not succeed in reaching stable acreage in France and does not meet the demand coming from users which incorporate linseeds, which display high content in omega 3 (alpha-linolenic acid), in animal feeding in order to deliver food enriched in omega 3 by natural way (dairy products, eggs, ham...). However, several driving forces should encourage this crop, like the shortfall in linseeds in the European Union and the environmental changes expected from agriculture. That is why the French oilseed crop organisation (Onidol) and the French technical institute for oilseed crops (Cetiom) launched in 2008 a study about the linseed situation in France. Aim of this work was to get a better understanding of each stage of the linseed chain production from the linseed cultivation to the users and to draw perspectives for the future of this crop in France. To do that a large survey was carried out according to methods adapted to each considered sector. A great attention was given to the progress of the variety offer because the yield gap between linseed and other crops in the rotation is probably one of the most important factors explaining the crop stagnation in France. The synthesis presented in the present paper allows identifying encouraging signals for the future and points the major drawbacks to overcome and the main advantages to take advantage from for developing linseed in France.
\end{abstract}

Key words: linseed, French production, supply chain, economic chain

d'un panorama actualisé de l'ensemble des maillons de la filière lin, des enquêtes ont été menées auprès de l'ensemble des opérateurs selon des techniques adaptées à chaque secteur. Une attention particulière a été portée sur la progression de l'offre variétale dans la mesure où le différentiel de rendement du lin oléagineux par rapport à d'autres cultures de l'assolement en France est probablement une des causes majeures de la stagnation de la culture. La synthèse des résultats de cette étude permet de tracer quelques perspectives encourageantes pour l'avenir de la production française de lin.

\section{Une source végétale inégalée en oméga 3}

Le lin oléagineux (Linum usitatissimum) est cultivé d'abord pour sa graine à la différence de son parent, le lin textile, sélectionné, lui, en priorité pour sa paille riche en fibres. Sa graine de petite taille (5 à $10 \mathrm{~g}$ pour 1000 grains) est riche en huile (38 à $40 \%$ pour une graine brute à $9 \%$ d'humidité) et moyennent dotée en protéines (22\%). Son originalité provient de son profil en acides gras dominé par la présence de l'acide alphalinolénique (C18:3 appartenant à la famille des oméga 3), à plus de $54 \%$ en moyenne du total (figure 1). L'usage de son huile a connu un fort développement en Europe au cours $\mathrm{du} x \mathrm{x}^{\mathrm{e}}$ siècle dans les secteurs non alimentaires. Ainsi, grâce à la forte siccativité et réactivité de son huile, le lin s'est avéré une matière première de choix pour la fabrication du linoléum (revêtement de sol), de peintures et d'encres mais aussi de savons. En parallèle les tourteaux, co-produits de I'huile lors de la trituration de la graine, ont connu un vif succès en alimentation animale et notamment auprès des éleveurs de bovins, probablement en raison de la teneur encore élevée en

Pour citer cet article : Labalette F, Landé N, Wagner D, Roux-Duparque M, Saillet E. La filière lin oléagineux française : panorama et perspectives. OCL $2011 ; 18(3)$ : 113-22. doi : 10.1684/ocl.2011.0383 


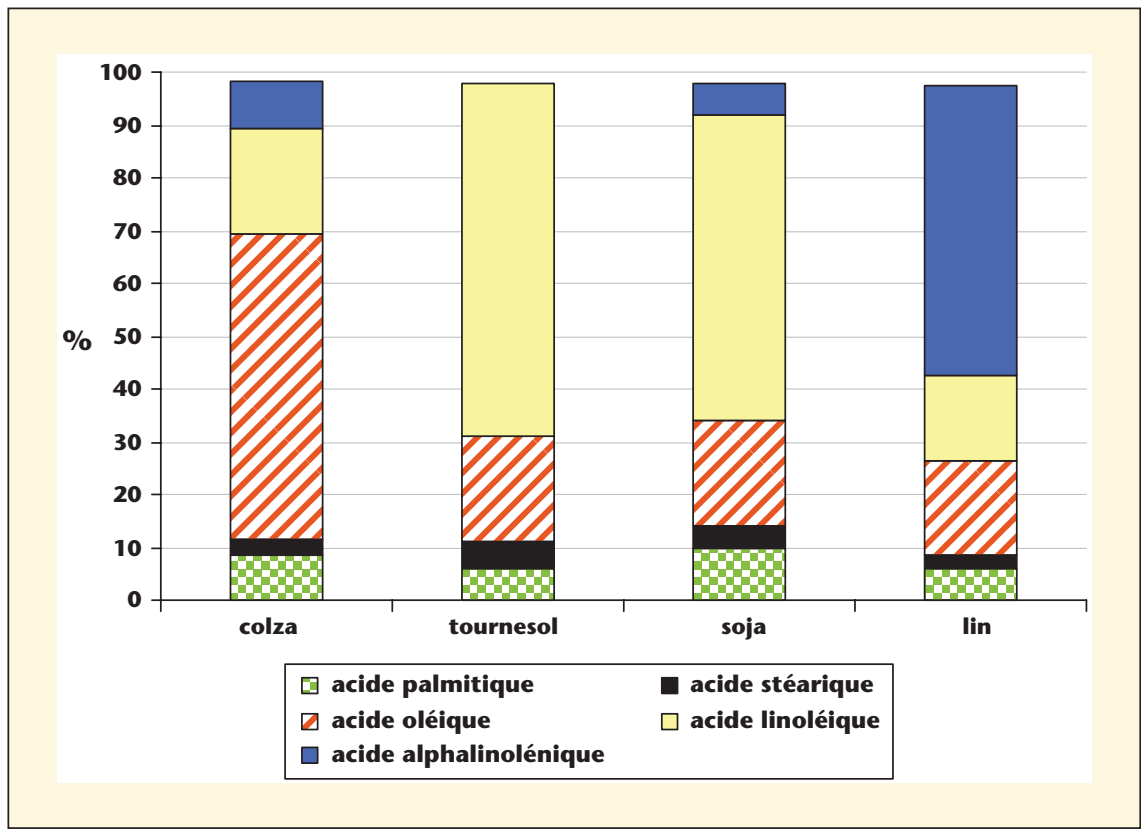

Figure 1. Profil en acides gras de I'huile de lin oléagineux en comparaison à d'autres huiles des pays tempérés. Source: Moyennes de données de I'ITERG.

matières grasses résiduelles (source d'énergie) et à la présence d'oméga 3 dont on ne connaissait pas explicitement les bienfaits.

Depuis une petite vingtaine d'années, la mise en évidence du rôle important sur la santé humaine de l'acide alphalinolénique et du déficit de cet acide gras dans les régimes alimentaires français (et de bien d'autres) ont donné un intérêt potentiel au lin en alimentation humaine. Cependant, sa forte teneur en acide alpha-linolénique en fait une huile délicate à conserver, et son utilisation d'huile reste très modérée en Europe même si celle-ci se développe un peu en consommation directe ou sous forme d'ingrédients pour les margarines par exemple. En France, et pour des raisons essentiellement liées à ces difficultés de conservation, I'huile de lin est restée longtemps interdite en alimentation humaine contrairement à la situation observée dans la majeure partie de I'Union européenne (décret du 12/02/73 - JORF 15/02/73 pp17301732 - article 3.2). Suite à une nouvelle évaluation (AFSSA, 2009), cet état de fait a évolué à la faveur de l'article $10 \mathrm{du}$ décret $n^{\circ}$ 2008-184 du 26 février 2008 et de l'arrêté du 4 décembre 2008 (Ministère de l'économie, 2008) qui autorise l'utilisation de I'huile de lin raffinée comme ingrédient en mélange dans les huiles alimentaires et les World 2010. poration de graines de lin dans leur alimentation. Un des industriels leaders de cette voie de valorisation est basé en France où il a contribué à construire une filière de qualité à vocation nutritionnelle partant de l'approvisionnement en graines jusqu'au consommateur en passant par l'extrusion de la graine de lin et la fabrication des aliments destinés aux élevages concernés. Cette filière, identifiée par un logo et structurée par la mise en place de cahiers des charges à chaque étape, a gagné une certaine reconnaissance de la part des pouvoirs publics (Lessirard, 2009). D'autres processus comparables d'enrichissement en oméga 3 de produits existent ailleurs mais ils n'ont pas forcément atteint ce même niveau d'organisation.

\section{Forte dépendance de I'Europe pour ses approvisionnement en graines}

matières grasses tartinables et encadre son niveau d'introduction via la teneur en acide alpha-linolénique de l'aliment considéré. Cette ouverture réglementaire n'a pas donné lieu à ce jour à une forte percée de l'huile de lin mais elle constitue un cadre favorable à d'éventuels travaux de R\&D en ce sens. En revanche, c'est sous forme de graines entières que s'est construite sa percée alimentaire au travers, d'une part, de la consommation directe dans des produits de boulangerie-viennoiserie et, d'autre part, de l'enrichissement de la teneur en oméga 3 de produits animaux via l'incor-
Le lin est une culture mineure au niveau mondial avec à peine $1 \%$ des surfaces totales d'oléagineux. Le principal pays producteur est traditionnellement le Canada (745 000 tonnes par an en moyenne sur la période 2000-2010) où le lin de printemps rentre dans les assolements des grandes plaines au grès des contextes de marché (figure 2). De I'autre côté de I'Atlantique, I'Union européenne est le premier utilisateur mondial de graines de lin, ses besoins fluctuant entre 500000 et 600000 tonnes par an (t/an) en premier lieu pour

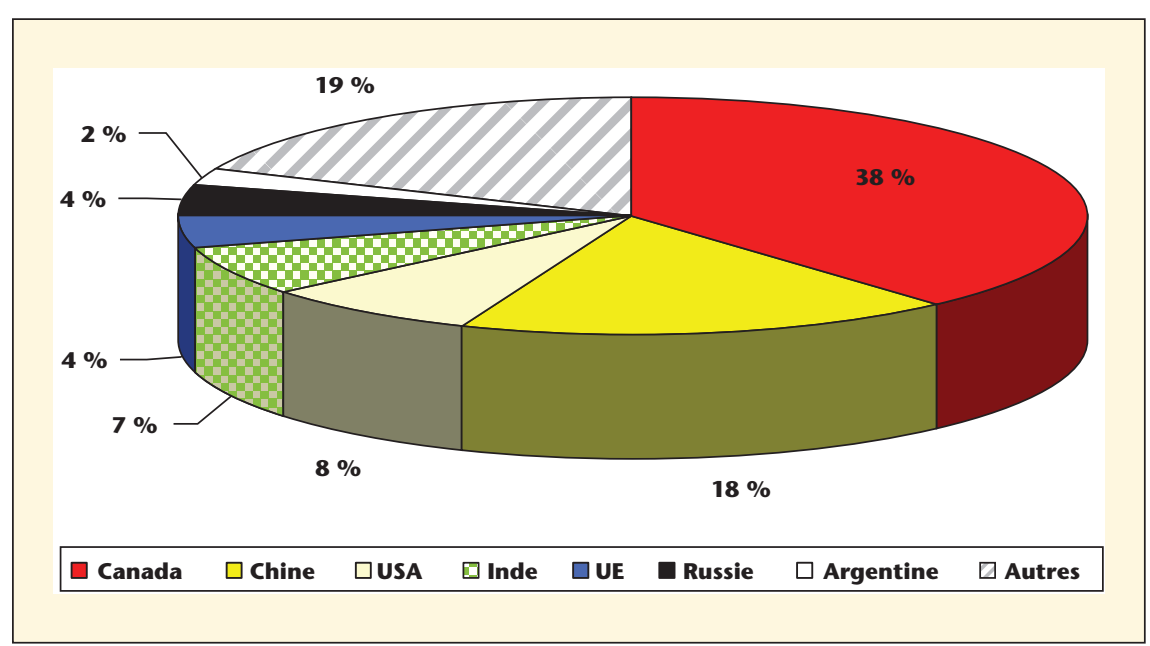

Figure 2. Principaux pays producteurs de lin oléagineux dans le monde en 2009. Source: Oil 
Tableau 1. Quantification des usages de la graine de lin oléagineux dans I'UE. Source: recoupements de données, Enquête Onidol 2008.

\begin{tabular}{|ll|}
\hline Utilisation & Tonnes de graines/an \\
\hline Trituration & 440 à 450000 \\
\hline Alimentation animale (graines entières) & 100 à 120000 \\
\hline Alimentation humaine (graines entières) & 30000 \\
\hline Total UE & 550000 à 600000 tonnes/an \\
\hline
\end{tabular}

approvisionner les unités de trituration concentrées au Benelux et en Allemagne (tableau 1) mais aussi pour couvrir les nouveaux usages alimentaires en graines entières.

Ne dépassant pas 4 à $5 \%$ de la production mondiale de graines de lin avec 80000 à 100000 t/an sur les trois dernières campagnes (figure 2), I'UE ne peut ainsi répondre qu'à $20 \%$ environ de ses besoins et se retrouve donc en situation de forte dépendance vis-à-vis des importations. Si son principal fournisseur reste le Canada, les États-Unis ont laissé la place à la Russie suivie de I'Ukraine au cours des dernières années, selon un mouvement similaire de développement des productions et exportations constaté pour d'autres oléagineux comme le tournesol (tableau 2).

La concentration des zones de production et de consommation, la forte dépendance aux importations en graines de I'UE, la faiblesse des volumes en jeu ainsi que la non-substituabilité d'un certain nombre d'usages rendent le marché des graines très sensible aux variations de la disponibilité en graines. Ainsi même si le cours des graines de lin suit plus ou moins les mouvements des oléagineux majeurs, en atteste le coefficient de corrélation de 0,57 avec le prix du soja, il peut aussi facilement s'en détacher, parfois à la baisse mais le plus souvent à la hausse, en réponse à tout événement susceptible d'entraîner une relative pénurie sur le marché (figure 3).

Cette volatilité des prix de la graine de lin constitue, on le verra, un facteur qui

pénalise le développement de la culture en Europe et en France.

\section{En France, une production de lin fluctuante qui peine à décoller}

Les surfaces en lin françaises occupent le deuxième ou troisième rang dans I'UE, derrière I'Angleterre (30 000 hectares [ha] en 2010) et la Belgique (14 000 ha en 2010). Affichant une moyenne annuelle de 12483 ha sur la période 1999-2010, le lin oléagineux a connu un premier pic à 36410 ha en 1994 suite à la réforme de la Politique agricole commune (PAC) de 1992 (prime pour les cultures non alimentaires sur jachère) puis un second à 18660 ha en 1999 à la faveur de l'aide à la culture du lin au cours des années 1999 et 2000 (figure 4). La forte décroissance observée ensuite sous I'effet de I'Agenda 2000, défavorable aux oléagineux dans leur contrecarrée par le développement de I'utilisation sous forme de graines de lin extrudées sur le territoire (années 2005 à 2007) avant de subir de plein fouet les conséquences de la hausse des prix agricoles de 2007 et 2008.

En termes de localisation des surfaces françaises, les bassins de production du lin oléagineux ont fortement évolué depuis les années 1990. Ainsi la Picardie, région traditionnelle de culture avec des variétés de lin de printemps, a perdu sa ensemble, a pu être temporairement

Tableau 2. Importation de graines de lin oléagineux dans I'Union européenne (en 1000 tonnes).

\begin{tabular}{|llll|}
\hline & $\mathbf{2 0 0 4}$ & $\mathbf{2 0 0 8}$ & $\mathbf{2 0 0 9}$ \\
\hline Canada & 445 & 437 & 408 \\
États-Unis & 50 & 40 & 1 \\
Russie & 20 (Russie et/ou Ukraine) & 49 & 82 \\
Ukraine & 5 & 9 & 28 \\
Autres & 520 & 22 & 12 \\
\hline Total & & 557 & 531 \\
\hline
\end{tabular}

place de leader (47\% des surfaces en 1994, $30 \%$ en 2000 et $12 \%$ en 2009) tandis que le Centre en 2000 puis l'Ouest-Atlantique en 2007 l'ont successivement supplantée jusqu'à atteindre un équilibre en 2009 avec environ $35 \%$ des surfaces françaises pour chacun de ces deux nouveaux bassins. On identifie principalement deux facteurs explicatifs de cette délocalisation des bassins de production du lin. L'arrivée des variétés de lin d'hiver (première inscription au catalogue français de la variété "Oliver» en 1995) permet la culture du lin oléagineux dans des régions plus exposées au stress hydrique estival du fait d'un plus faible potentiel des terres : région Centre, région PoitouCharentes, région Pays-de-Loire, région Bourgogne. .. Et l'émergence du marché graines entières grâce à un acteur économique basé en Bretagne a probablement fini $d$ 'impulser ce mouvement de relocalisation de la culture au sud de la Seine.

\section{Des rendements au champ qui évoluent moins vite que le progrès génétique}

Au champ, le rendement du lin oléagineux moyen sur la période 2006-2009 s'établit selon la source statistique Agreste à 20,9 quintaux/ha ( $q / h a)$. Les résultats de l'enquête postale et téléphonique, menée auprès de 124 producteurs de lin en France dans le cadre de la présente étude, confirment ce chiffre avec un rendement moyen de l'échantillon de $21 \mathrm{q} /$ ha sur la même période 2006-2009 et mettent en évidence l'absence de différence significative entre lins de printemps et hiver (au sein de l'échantillon). L'enquête apporte une information complémentaire sur la variabilité des rendements : $10 \mathrm{q} / \mathrm{ha}$ entre les valeurs extrêmes.

Contrairement à ce que I'on pourrait croire, ce niveau relativement faible de rendement ne semble pas imputable au potentiel des variétés. En premier lieu, le lin oléagineux bénéficie d'un effort de sélection remarquable au regard des surfaces françaises et européennes avec la présence active de trois semenciers sur le territoire national. Les possibilités de mutualisation avec les programmes consacrés au lin textile en sont probablement l'explication majeure. La très 


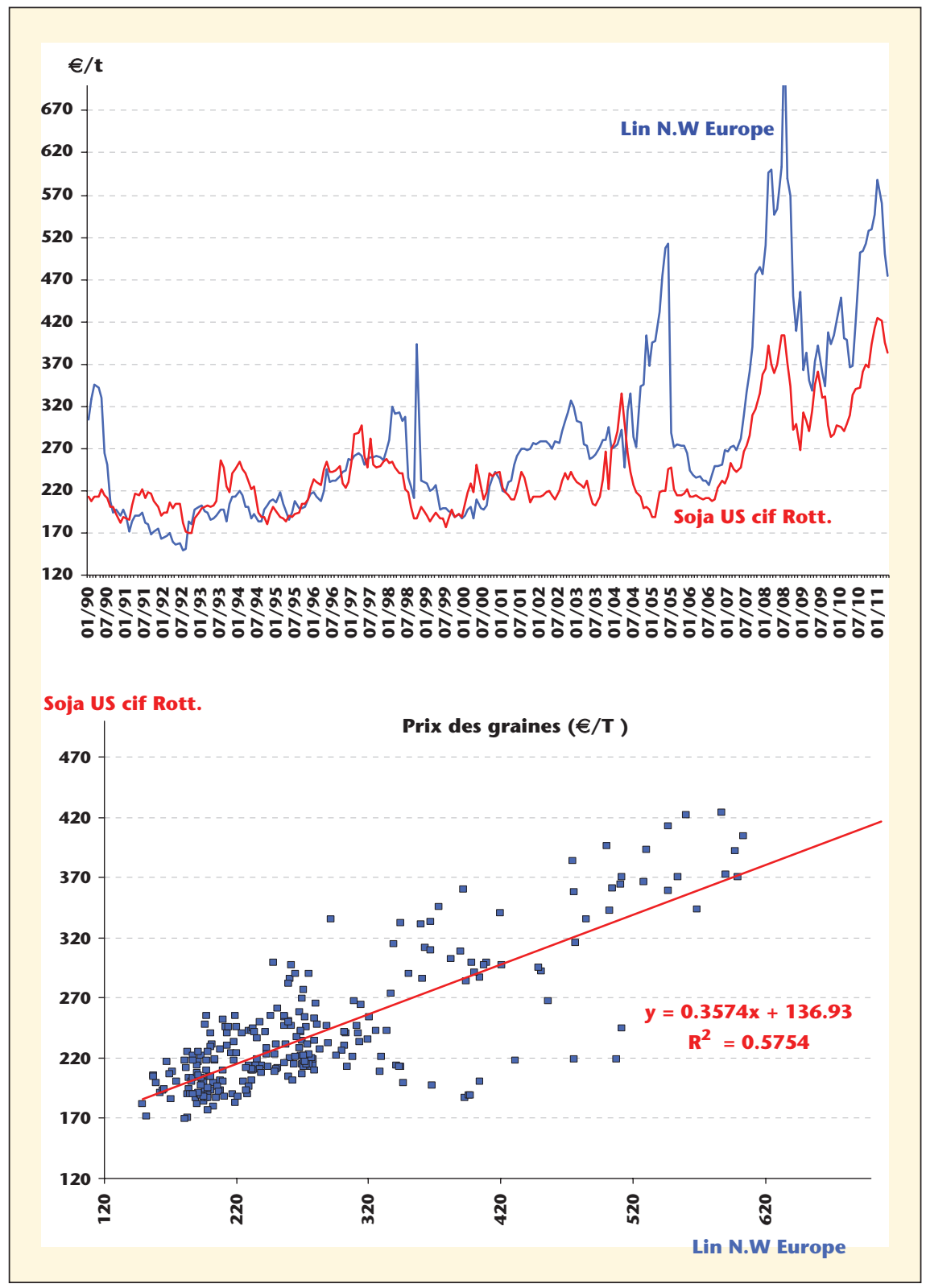

Figure 3. Évolution du cours de la graine de lin oléagineux en regard de celle de soja (Source données : Oil World).

faible utilisation de semences fermières malgré le caractère autogame de l'espèce constitue aussi un atout non négligeable pour renouveler les variétés auprès des agriculteurs. II en résulte une offre variétale diversifiée avec pas moins de 28 variétés inscrites au catalogue français 2010 (20 de type printemps et huit de type hiver) dont certaines se distinguent par une très haute teneur en acide alphalinolénique (Lutéa par exemple). En second lieu, une amélioration de la productivité des variétés a pu être mise en évidence par l'étude conduite par l'Onidol et le Cetiom sur les variétés inscrites au catalogue français. Ce travail a été basé sur les données brutes de rendement de ces variétés mesurées dans les essais des deux années d'examen du Comité technique permanent de la sélection (CTPS). Ces données ont été exprimées ensuite sous forme d'un indice par rapport à un témoin constant (Barbara pour le lin de printemps et Oliver pour le lin d'hiver) et replacées sur l'année d'inscription de la variété considérée (figures 5 et 6). Une régression linéaire est faite à partir des moyennes annuelles des indices des variétés inscrites (si l'on a une seule variété inscrite, la moyenne est alors égale à l'indice de la variété). Le progrès génétique en rendement ( $q /$ ha/an) est calculé sur la période en multipliant le rendement $(q / h a) d u$ témoin sur la période considérée par le coefficient directeur de la droite de régression ramené en pourcentage (soit $0,3574 \%$ pour le lin d'hiver et 0,2048\% pour le lin de printemps).

Le progrès génétique a ainsi été évalué par cette méthode à 0,44 q/ha/an pour le lin de printemps, ce qui est loin d'être négligeable et s'avère peu éloigné de valeurs observées sur d'autres cultures 


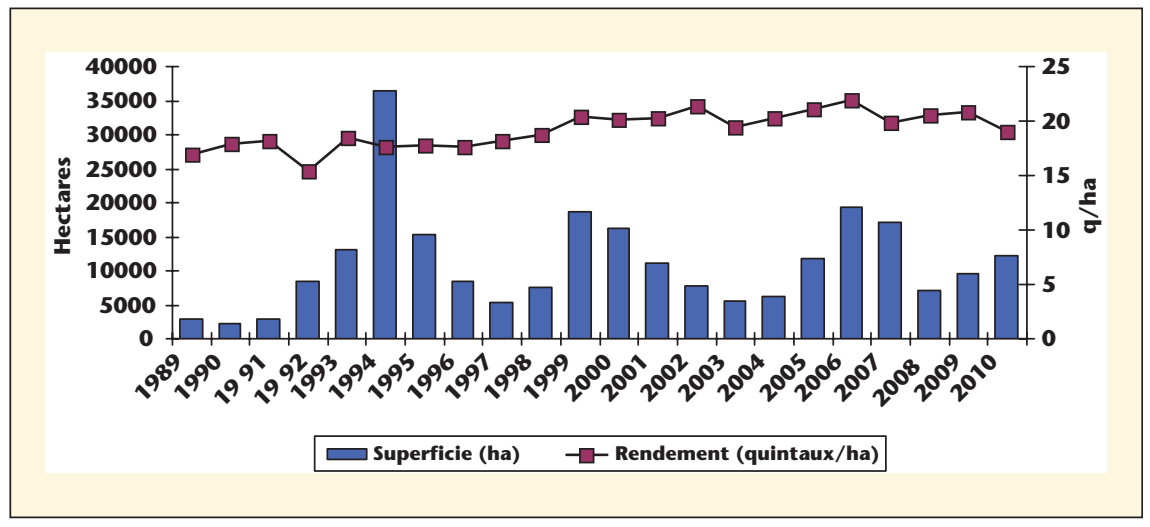

Figure 4. Évolution des surfaces et rendements de la culture du lin oléagineux en France. Source: Agreste mai 2009 sauf pour surfaces 2010 (FranceAgriMer, septembre 2010).

comme le soja en France. En lin d'hiver, le résultat plus élevé de $0,76 \mathrm{q} / \mathrm{ha} / \mathrm{an}$ doit être nuancé par le faible nombre de variétés de l'échantillon étudié et par le démarrage récent de la sélection.

Cette augmentation des rendements ne se retrouve pas dans les mêmes proportions au champ. Le gain est évalué ici à $0,2 \mathrm{q} / \mathrm{ha} / \mathrm{an}$ depuis 1970 , sans distinction entre types d'hiver et printemps. Il existe donc un écart entre la génétique et les résultats en plein champ que les agriculteurs comme les organismes collecteurs interrogés relèvent implicitement en évoquant des niveaux de rendement insuffisants et irréguliers (figure 7).

Les conséquences de ce différentiel de progression du potentiel entre l'évaluation des variétés à leur inscription et leurs performances observées chez les agriculteurs se retrouvent dans les rendements obtenus ces cinq dernières années. De 2006 à 2010, le rendement moyen dans les essais du réseau officiel d'évaluation des variétés (CTPS) s'établit autour de 23,8 q/ha (écart-type $1,2 \mathrm{q} / \mathrm{ha}$ ). À l'inverse, le rendement moyen en France, pour cette période, se chiffre autour de 20,2 q/ha (écarttype $1 \mathrm{q} /$ ha, Agreste, 2011).

Plusieurs facteurs ont été identifiés pour expliquer à la fois cette moindre progression et cette moindre performance en production dans la plaine française. Les variétés évaluées à l'inscription sont renouvelées chaque année (à l'exception des témoins), souvent plus performantes, a contrario, les moyennes françaises prennent en compte l'ensemble des variétés cultivées des plus

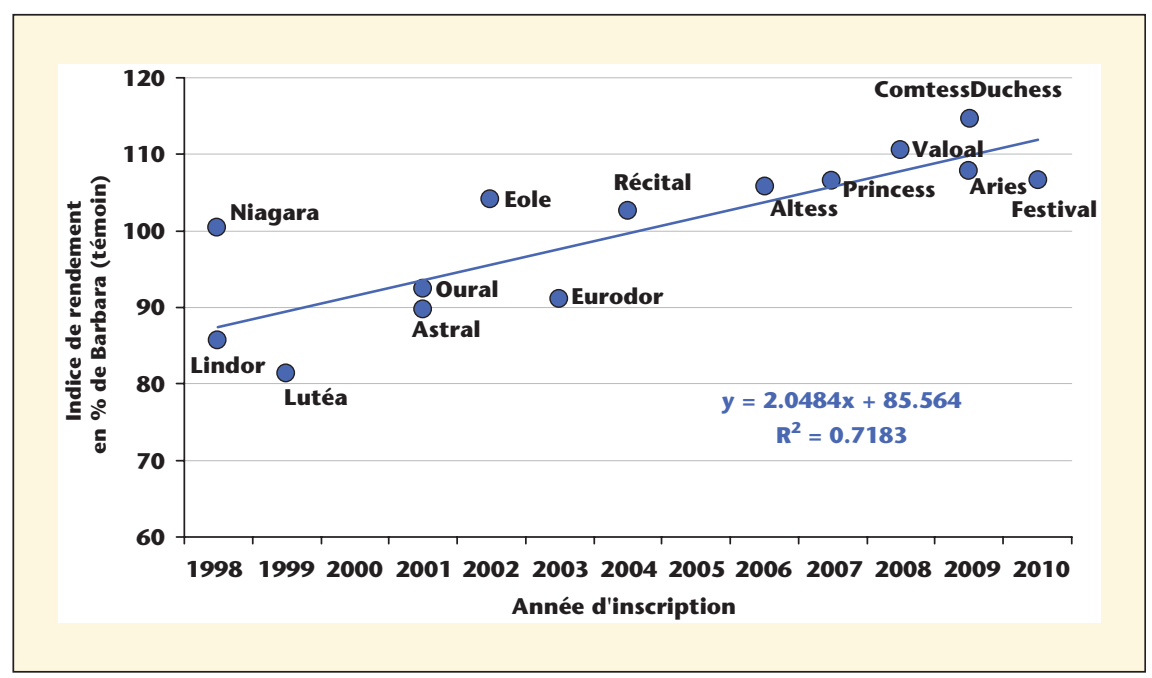

Figure 5. Estimation du progrès génétique sur le facteur rendement en lin oléagineux de printemps en France d'après les données des essais du CTPS (étude Onidol/Cetiom 2010). anciennes aux nouvelles. D'après Semences \& Progrès (Collectif, 2010), si I'on considère que les surfaces en multiplication sont les variétés semées compte-tenu du faible taux de graine de ferme utilisé (selon notre étude), les surfaces en multiplication de 2006 à 2009 montrent que les variétés, inscrites au catalogue français, restent emblavées pendant une dizaine $d$ 'années. Ce faible taux de renouvellement des variétés est un des premiers facteurs pouvant expliquer la lente arrivée du progrès génétique dans les parcelles.

Un autre phénomène identifié au cours de cette étude et pouvant participer à l'explication de ces écarts entre les progrès de la génétique et la réalité de la plaine française est l'arrivée massive des lins d'hiver depuis ces cinq dernières années. Ceux-ci présentent des performances supérieures d'après leurs rendements moyens entre 2006 et 2010 (données d'inscription CTPS) comparés aux rendements des lins de printemps récemment inscrits sur cette même période, soit $24,7 \mathrm{q} /$ ha contre $22,9 \mathrm{q} / \mathrm{ha}$. Cependant, une caractéristique agronomique du lin d'hiver est de pouvoir être implanté en milieu moins favorable (sols superficiels, conditions hivernales plus froides, été plus échaudant) que les lins de printemps. Malgré des performances plus importantes, ces environnements permettent difficilement de mettre en valeur le lin d'hiver. Aujourd'hui, la sole française est emblavée à $80 \%$ de lin d'hiver et hors du bassin traditionnel, tempéré et à sol profond de la Picardie (selon notre étude).

Enfin, le dernier facteur pouvant être retenu pour expliquer ces écarts de performances entre un potentiel génétique et la réalité en parcelle agricole est d'ordre technique. Notre étude a montré une certaine méconnaissance de la culture conduisant à des pratiques non adaptées voire pénalisantes. On peut citer les densités de semis non adaptées, I'usage de régulateur de croissance, les limites du désherbage chimique, les contraintes de la récolte, voire la mauvaise gestion de l'azote...

Au final, le lin, qui doit s'entendre comme une culture technique requerrant un suivi régulier (CETIOM, 2006 ; CETIOM, 2007), mériterait sans doute un accompagnement et un intérêt technique plus poussés de la part des organismes économiques et de dévelop- 


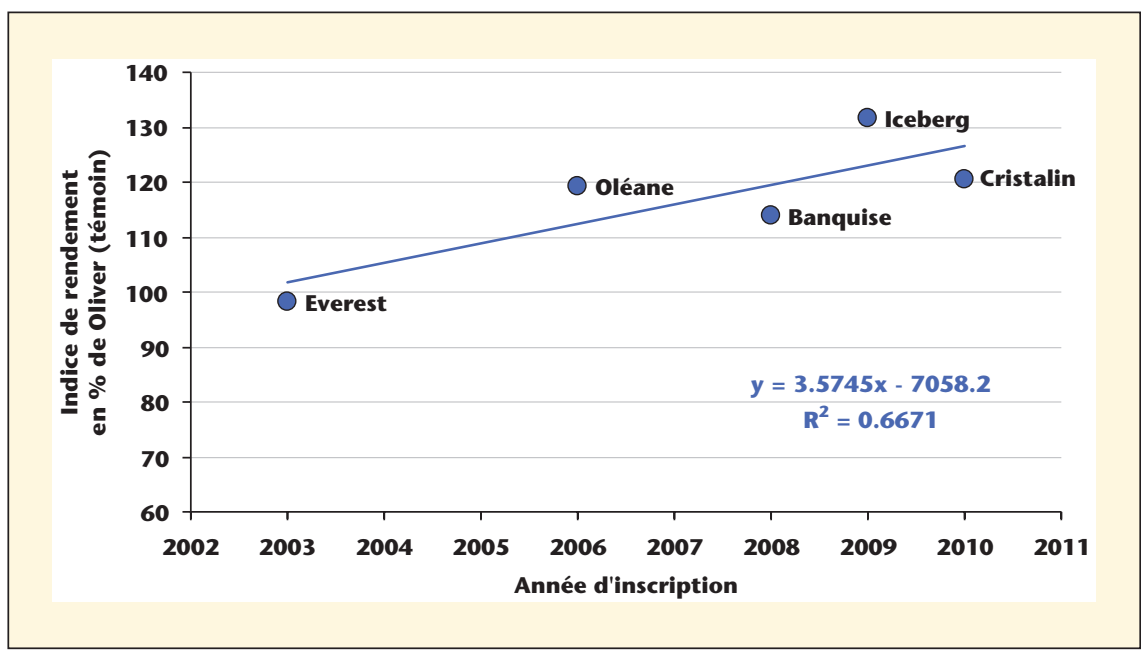

Figure 6. Estimation du progrès génétique sur le facteur rendement en lin oléagineux d'hiver en France d'après les données des essais du CTPS (étude Onidol/Cetiom 2010).

pement pour gagner la confiance des agriculteurs.

\section{Une offre variétale répondant aux exigences de qualité de I'aval}

En termes de qualité de la graine, la méthode d'analyse utilisée pour la productivité et appliquée à la teneur en huile des variétés inscrites au catalogue français montre qu'aucun progrès significatif n'a été réalisé sur ce facteur en raison probablement d'un effort de sélection faible sur ce critère. Cela n'empêche toutefois pas d'observer des différences entre variétés et entre années, les teneurs en huile pouvant descendre autour de $38 \%$ pour une graine à $9 \%$ d'humidité (référence d'usage en France établie par analogie au colza ou au tournesol) et s'élever à près de $45 \%$. Pour ce qui concerne la teneur en acide alphalinolénique, les variétés de lin commercialisées présen-

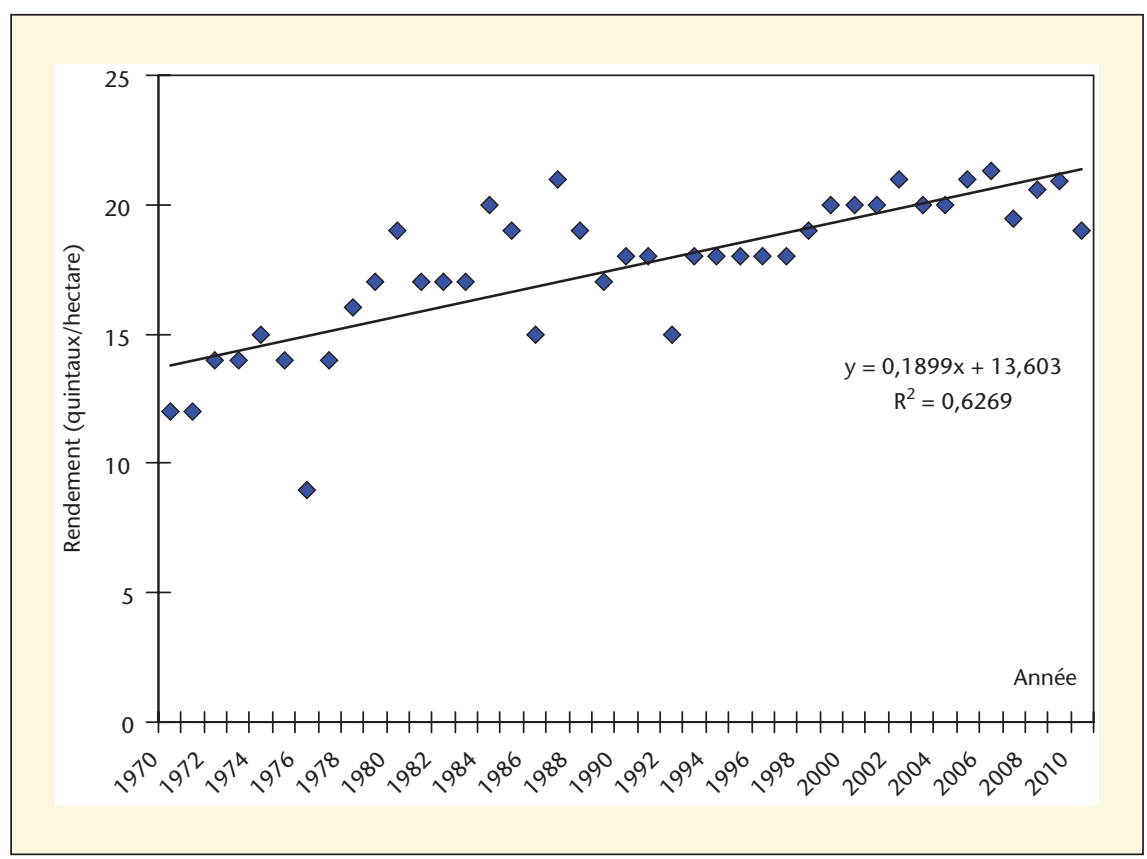

Figure 7. Évolution des rendements "agriculteurs» en graines du lin oléagineux en France. Source: AGRESTE, mai 2011. tent une gamme de variation importante et à même de répondre aux besoins des utilisateurs. La caractérisation menée en 2005 a montré que l'effet génétique était fort avec environ 10 points d'écart entre la plus basse $(51,5 \%)$ et la plus forte teneur $(66,2 \%)$ chez le lin de printemps (Travaux Cetiom-Onidol-Obtenteurs, en 2005). L'effet lieu n'était pas en reste, notamment pour le lin de printemps avec une différence de près de 14 points entre le lieu le plus faible $(48,7 \%)$ et le plus élevé (63\%), toujours en 2005. Chez le lin d'hiver, l'effet lieu semblait atténué, observation qui serait à confirmer avec des localisations plus proches des lieux réels de culture, les opérateurs ayant exprimé leur intérêt pour cette question. De manière générale, les lins d'hiver, maintenant majoritaires en production, se distinguent par une teneur en oméga 3 plus élevée en moyenne que les lins de printemps ( 3 à 4 points de mieux dans l'étude Cetiom-Onidol-Obtenteurs de 2005 et 2006). Parmi les variétés actuelles, beaucoup dépassent en moyenne $55 \%$ qui constitue le minimum exigé contractuellement en France et les cahiers des charges pratiqués comportent souvent une liste variétale recommandée prenant en compte ce critère. D'ailleurs, l'évolution confirmée vers un marché de qualité centré sur la valorisation des oméga 3 conduit à l'introduction progressive de la mesure de la teneur en acide alphalinolénique dans l'évaluation des variétés lors des procédures d'inscription et de post-inscription. Tout cela ne peut qu'encourager les obtenteurs à garder la pression sur ce critère avec en ligne de mire une cible autour de $60 \%$.

\section{Une culture de diversification d'intérêt à l'échelle de la rotation}

Les 124 agriculteurs questionnés par le Cetiom en 2009 (soit $11 \%$ de la sole de lin en 2009) mentionnent la diversification et l'allongement des rotations au premier rang des atouts de la culture du lin. La comparaison des 93 rotations sans lin avec les 90 rotations avec lin de l'échantillon montre que la présence du lin permet de diminuer les rotations courtes (de moins de quatre ans) de $20 \%$ et au contraire allonge leur durée 
(six ans et plus) sans se substituer à une autre culture dans $42 \%$ des situations. Dans l'échantillon enquêté par voie postale et téléphonique, la surface de lin moyenne mise en culture est d'environ 13 à 16 ha par exploitation soit $8 \%$ de leur assolement. Globalement, cet effet de diversification est mentionné par l'ensemble des opérateurs rencontrés notamment les semenciers et les organismes stockeurs.

Autre avantage, souligné par une majorité de ces agriculteurs, le lin ressort comme relativement économe en intrants. Une bonne illustration en est donnée par l'analyse des données technico-économiques des cultures pratiquées de 2004 à 2009 par dix producteurs du groupement Graine de Lin 28 sur les mêmes parcelles (à conditions pédoclimatiques identiques). L'économie de charges opérationnelles observée pour le lin est d'environ 100 euros par hectare (€/ha) par rapport au blé ou au colza en raison notamment de moindres apports en fertilisants azotés et en fongicides (tableau 3).

Cependant, malgré des charges moins élevées, la marge brute du lin apparaît dans les conditions de l'étude en retrait de $193 € /$ ha par rapport à celle d'une autre tête d'assolement comme le colza, cela en raison de revenus plus faibles liés à des rendements insuffisamment compensés par les prix. Cette moindre performance économique et surtout la fluctuation des revenus que I'on peut attendre de la culture arrivent d'ailleurs en deuxième position des freins cités au développement du lin dans l'enquête du Cetiom en 2009 auprès des 124 agriculteurs. Outre évidemment ces deux leviers rendements et prix que I'on doit tenter d'activer si l'on souhaite pérenniser cette filière, il faut examiner les résultats à l'échelle de la rotation pour mieux appréhender les termes possibles de la compétitivité du lin. La comparaison des marges brutes du blé en fonction de trois précédents différents (blé, colza et lin) est éloquente. Le gain observé pour un blé avec précédent lin varie de 100 à $200 €$ /par rapport à un précédent colza ou blé par un double effet d'augmentation du rendement du blé suivant et de la baisse des intrants nécessaires au blé suivant (figure 8). Ainsi, le déficit de marge brute qui freine sans doute le lin comparativement aux autres cultures majeures de la rotation s'en trouve globalement réduit. Mais, pour gommer le déficit restant et rendre la culture compétitive à court terme, le rapport de prix par rapport au colza ou au blé demanderait à être un peu renchéri.

Enfin, au-delà des aspects économiques, les baisses d'intrants consécutives à l'introduction du lin contribuent à améliorer les impacts environnementaux de la succession de cultures. C'est donc résolument à l'échelle de la rotation que le lin devrait trouver sa meilleure justification, y compris pourquoi pas au travers de dispositifs de soutien reconnaissant ce service rendu à I'environnement.

\section{Une collecte dispersée mais orientée vers la qualité}

L'enquête menée par l'Onidol au cours du deuxième semestre 2009 dans les trois principaux bassins de culture (Ouest-Atlantique, Centre et Picardie) de lin oléagineux a permis d'obtenir de I'information sur deux années de récolte (2008 et 2009) ainsi que sur les perspectives 2010. Réalisée auprès de 20 collecteurs de lin, elle se caractérise par une très bonne représentativité

Tableau 3. Données technico-économiques moyennes des trois principales cultures pratiquées par dix agriculteurs du Groupement Graine de lin 28, sur une période de cinq ans (données calculées à partir des pratiques réelles des agriculteurs de 2004 à 2009, des prix des intrants issus de la base de données d'Agrotransfert Picardie et de la Chambre d'agriculture de l'Eure-et-Loir, et des prix de vente réels des agriculteurs enquêtés, moyennés sur les cinq ans pour s'affranchir des fluctuations annuelles)-Cetiom, 2009.

\begin{tabular}{|llll|}
\hline Jeu de données & Blé & Colza & Lin \\
\hline nombre de données & 26 & 13 & 9 \\
rendement moyen $(\mathrm{t})$ & 8,3 & 3,6 & 2,3 \\
prix moyen $(€ / \mathrm{t})$ & 154 & 276 & 316 \\
charges op. $(€ / \mathrm{ha})$ & 418 & 371 & 280 \\
marge brute $(€ / \mathrm{ha})$ & 860 & 623 & 447 \\
\hline
\end{tabular}

générale et par bassin, avec respectivement $86 \%$ pour la récolte 2008 et $71 \%$ pour 2009 des surfaces prises en compte. A contrario ce grand nombre d'opérateurs enquêtés pour un nombre $\mathrm{d}$ 'hectares limité révèle la grande dispersion de la production et peut-être une certaine frilosité à s'engager sur cette culture. Difficile en effet de consacrer des moyens techniques et de promotion significatifs lorsque le lin compte pour 0,5 à $1,7 \%$ de la collecte totale en graines de l'organisme stockeur soit 300 à 350 ha en moyenne. Qui plus est, les opérations de logistique et de stockage ne sont pas sans contrainte en raison notamment de la fluidité de la graine (les graines " coulent") qui engendre des bennes mal remplies et des coûts de stockage élevés (pas de stockage à plat possible). En revanche, le séchage ainsi que le nettoyage de la graine sont rarement nécessaires. La production s'avère aujourd'hui être tracée et sous cahier des charges pour environ deux tiers de la collecte et $s^{\prime}$ oriente de plus en plus vers les filières de valorisation exigeantes en qualité. La ventilation des débouchés l'illustre bien puisque le plus important d'entre eux, à près de $90 \%$ en moyenne sur les trois dernières années, s'avère être I'utilisation en graines entières dans le cadre de filières animales de qualité reposant sur l'enrichissement en oméga 3 des produits délivrés (figure 9). Le débouché graines entières en alimentation humaine (5 à $6 \%$ de la collecte enquêtée) est lui encore plus contraignant en termes de cahier des charges mais offre en contrepartie une meilleure valeur ajoutée alors que la trituration, elle, apparaît comme un marché d'opportunité lié aux fluctuations de disponibilité des graines d'importation.

La mise en marché s'effectue selon des voies diverses avec pour plus de $40 \%$ du volume l'intervention de structures intermédiaires de courtage ou de type associatif (OléoLin, Lin tradition ouest, Grain du Val-de-Loire) auxquelles se rajoutent les groupements de producteurs. Agissant à un échelon local, ces interfaces répondent à un besoin de stimuler et organiser l'offre souvent insuffisante et très dispersée vis-à-vis des utilisateurs et parfois même elles jouent un rôle dans l'accompagnent technique de la production. En ce sens, elles compensent probablement le relativement faible investissement sur le lin 


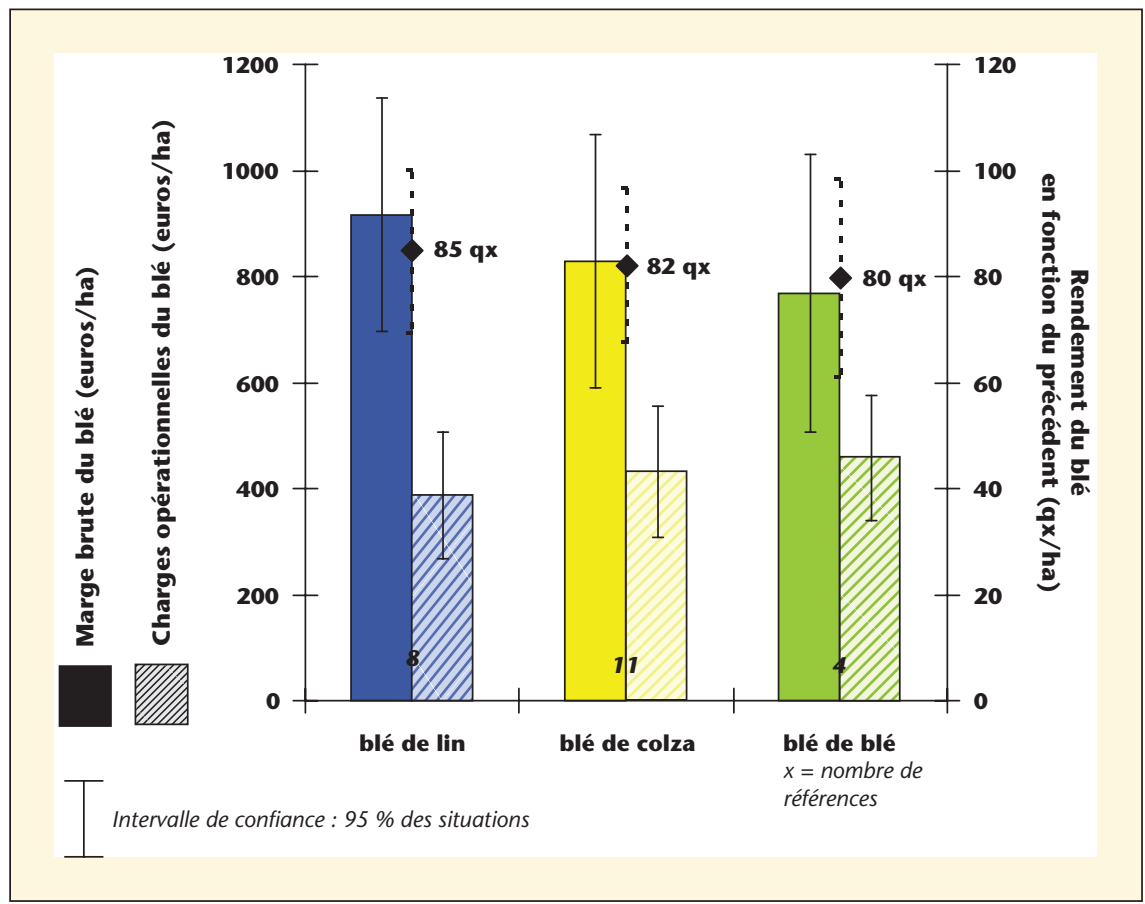

Figure 8. Marge brute, charges opérationnelles et rendement du blé en fonction du précédent. Étude de dix exploitations du groupement Graine de lin 28, période cinq ans (données calculées à partir des pratiques réelles des agriculteurs de 2004 à 2009, des prix des intrants issus de la base de données d'Agrotransfert Picardie et de la Chambre d'agriculture de l'Eure-et-Loir, et des prix de vente réels des agriculteurs enquêtés, moyennés sur les cinq ans pour s'affranchir des fluctuations annuelles). Source : Cetiom, 2009.

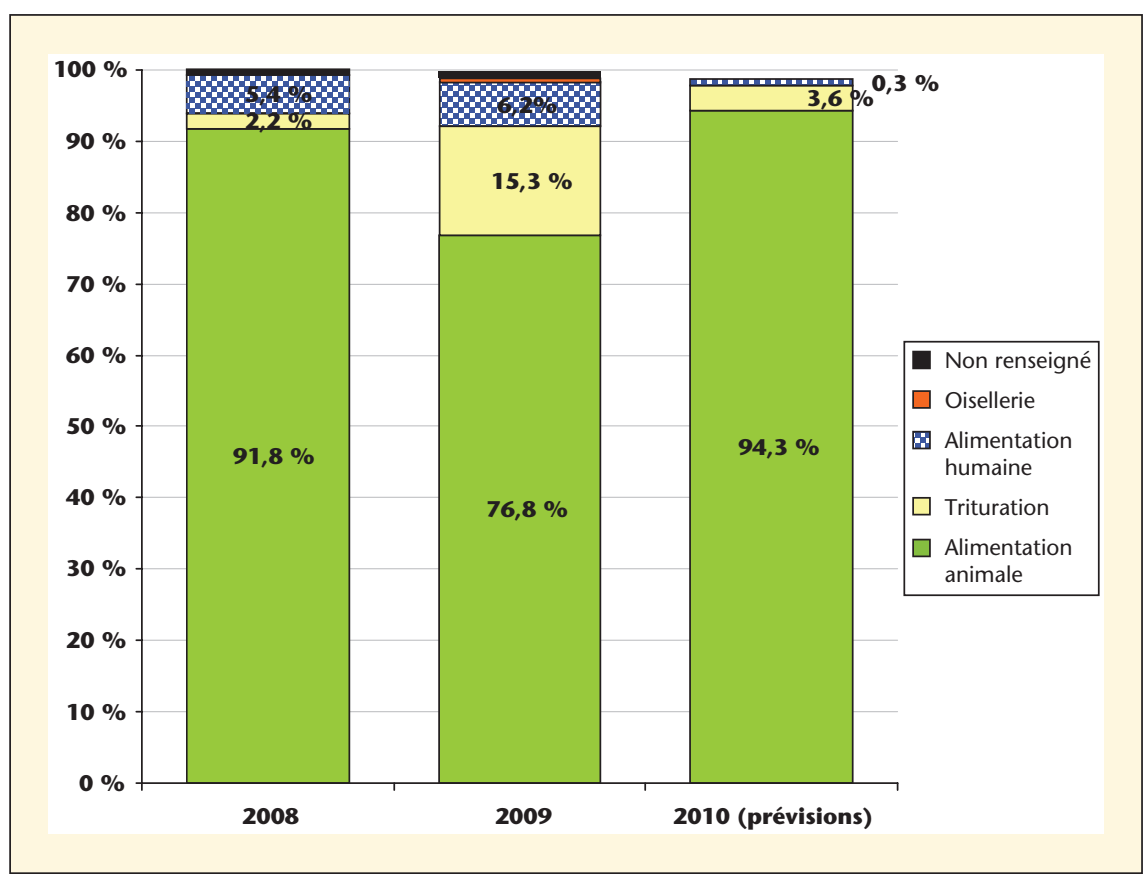

Figure 9. Répartition des débouchés des graines de lin issues de la collecte enquêtée en 2009 par l'Onidol. d'acteurs majeurs comme les organismes stockeurs et de développement. Au niveau des conditions de mise en marché, l'évolution vers un système contractuel plus sécurisé est résolument en marche avec I'implication forte d'opérateurs d'amont et du principal utilisateur de graines entières (Valorex). D'un prix fixe peu adapté à la grande volatilité des prix agricoles observée depuis 2007, les contrats passent progressivement à des systèmes prenant en compte les prix d'une ou plusieurs autres cultures présentes dans I'assolement. On trouve par exemple des rémunérations basées sur le cours moyen d'un panier de cultures (blé, colza, lin) au cours de la campagne considérée et encadrées par un prix minimum (environ 350 euros par tonne lors de l'enquête en 2009) et un prix maximum (environ 450 euros par tonne, prix probablement revu à la hausse autour de 480 à 500 euros par tonne pour la campagne 2010-2011). En Angleterre, le système pratiqué pour la filière qualité repose sur le prix du colza MATIF auquel s'ajoutait en 2008 et 2009 un complément « lin " d'environ $70 € /$ tonne. De surcroît, les contrats prévoient une prime à la teneur en oméga 3 pour les lots ayant une teneur supérieure ou égale à $56 \%$ et pénalisent ceux qui sont inférieurs à $54 \%$. Le montant du bonus/malus est croissant au fur et à mesure que l'on s'éloigne de cette valeur seuil et atteint de 3 à $7 € /$ tonne dans la gamme comprise entre 56 et $60 \%$. Aujourd'hui, ces efforts pour sécuriser les revenus des agriculteurs et les approvisionnements en termes de prix et d'organisation doivent être poursuivis et gagneraient sans doute à être harmonisés, le marché français souffrant encore de comportements d'opérateurs parfois contradictoires.

\section{Des utilisations industrielles supérieures à l'offre en graines françaises}

Trois principaux utilisateurs sont recensés sur le territoire français. Le premier, évoqué plus haut, pratique l'extrusion de graines entières à destination de I'alimentation animale pour un besoin total annoncé de 35000 tonnes par an en 2009. Cet opérateur est à l'origine de la création de la filière la plus visible de valorisation des oméga 3 en vue de I'alimentation humaine via 


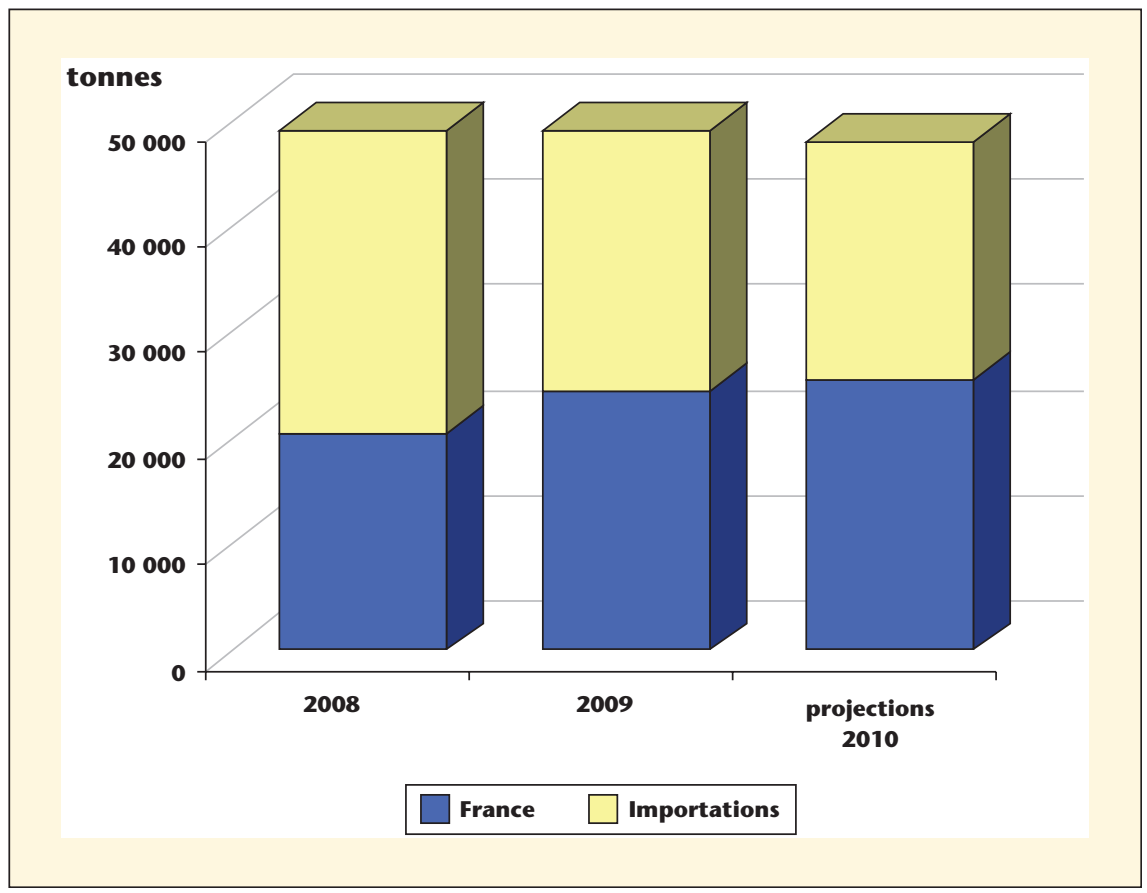

Figure 10. Origine des approvisionnements en graines de lin des utilisateurs français enquêtés en 2008 et 2009 par l'Onidol.

I'enrichissement des produits animaux. Celle-ci repose sur un cahier des charges et un affichage (logo Bleu-Blanc-Cœur) partagés par les intervenants $d$ 'amont et d'aval jusqu'à la grande distribution et sa promotion est étayée par le résultat d'études spécifiquement conduites sur le lin en France. Si I'ensemble des graines de lin achetées par cet industriel n'est pas engagé dans cette filière de qualité, une part croissante y est affectée avec un effet dynamisant sur les fournisseurs de graines. Le second en volume est un petit triturateur historique localisé en Picardie qui écrase en moyenne 10 à 12000 tonnes de graines de lin par an pour les marchés traditionnels de l'huile (export dans I'UE en grande majorité) et du tourteau gras pour l'alimentation animale. Le dernier est spécialisé dans la fourniture d'ingrédients au secteur agroalimentaire sous forme de graines seules ou en mélange prêtes à l'emploi en boulangerie et biscuiterie. Cet opérateur consomme moins de graines de lin (environ 2000 tonnes par an en 20082009, Enquête Onidol 2009) mais l'ensemble de son approvisionnement est soumis à un strict cahier des charges auprès de fournisseurs sélectionnés (beaucoup d'agriculteurs en direct) en France ou en Angleterre. Au total, le marché français s'élève donc à environ 50000 tonnes de graines de lin par an, redéploiement des surfaces à hauteur d'au moins 20000 ha d'ici 2015 est possible à condition de pérenniser des modalités de rémunération des producteurs de graines suffisamment incitatives. Tout d'abord, le lin, on l'a vu, constitue une solution de diversification d'intérêt dans un mouvement inéluctable de baisse des intrants auquel il peut contribuer. Plus en aval, son introduction dans I'alimentation des vaches laitières pourrait aussi apporter un service environnemental sous la forme de la baisse des émissions de méthane (Brunschwig et al., 2010) dont le processus de quantification et de reconnaissance par les pouvoirs publics a été engagé (travaux en cours sous la houlette de Valorex). Au niveau des marchés, la demande sociétale en faveur d'aliments bons pour la santé et pour l'environnement pourrait progressivement faire décoller la consommation de produits animaux naturellement enrichis en oméga 3 via l'alimentation du bétail, avec l'introduction du lin mais aussi d'autres sources d'oméga 3. Ainsi, les opérateurs français tableraient sur un besoin d'environ 50000 tonnes de graines de lin oléagineux tracé sous cahier des charges d'ici à 2012-2013 (CarluerLorssoruan, 2009). Additionnée aux autres débouchés, modestes certes, identifiés plus haut, cette demande pourrait justifier aux mêmes échéances une augmentation des surfaces de lin sous contrat à environ 20000 ha en France.

À cela pourraient s'ajouter à un horizon de cinq à dix ans de nouvelles utilisations, toujours sous-tendues par des objectifs nutritionnels d'amélioration de l'équilibre en acides gras, comme ingrédients dans le secteur de l'alimentation humaine. Les incorporations sous forme d'huile pourraient augmenter à condition de parvenir à bien en maîtriser le raffinage et les farines de lin, dont la fabrication démarre à peine, pourraient éveiller l'intérêt de I'industrie agro-alimentaire. En parallèle, l'oléochimie ouvre à moyen terme aussi des perspectives de valorisation, notamment au travers de la synthèse de polymères biosourcés pour lesquels une recherche active est en cours en Europe chez certains industriels.

\section{Un potentiel de 20000 hectares à court terme}

L'ensemble des éléments de contexte concourt à ce stade à considérer qu'un
De plus la paille de lin, qui est aujourd'hui considérée par les acteurs interviewés aussi bien comme un frein en raison de sa gestion un peu délicate (cas de $15 \%$ des agriculteurs, enquête Cetiom 2009) que 
comme un atout (valorisations en litières, paillage, isolants dans un tiers des situations enquêtées) pourrait à court et moyen terme se convertir définitivement en coproduit d'intérêt. Des travaux et expériences menées dans diverses régions montrent son potentiel comme matière première pour la fabrication de biomatériaux, en particulier de panneaux isolants. Une des difficultés majeures réside probablement dans la faible productivité en paille par hectare de la culture (à peine 2 tonnes collectables par hectare). Pour qu'un développement sérieux émerge, il faudra s'appuyer sur des initiatives industrielles locales de transformation de fibres végétales dont le lin serait une des composantes. Les conditions techniques (compromis entre productivité par hectare en paille et prévention de la verse au travers de l'application de régulateurs de croissance par exemple), logistiques et économiques permettant une juste rémunération du produit auprès des agriculteurs restent encore à préciser. II faut sans doute s'y préparer en saisissant les opportunités de proximité pour que le lin participe à sa mesure à la fourniture des futurs marchés des biomatériaux en Europe attendus en forte croissance.
Il n'est alors pas interdit grâce à ce faisceau de nouveaux usages $d^{\prime}$ 'imaginer qu'une deuxième étape de redéploiement des surfaces puisse advenir, avec une cible d'environ 30000 hectares de lin en France.

Répondre à ces marchés immédiats ou de plus long terme implique un maintien des efforts engagés en création variétale, en maîtrise technique de la culture ainsi que dans l'organisation $d^{\prime}$ une filière de production de qualité. Adossés à une mobilisation mieux concertée et mieux partagée entre l'ensemble des acteurs du lin français et à une implication proportionnée des organismes interprofessionnels de la filière oléagineuse, ils devraient contribuer à faire du lin oléagineux une culture de diversification durablement présente dans les assolements français.

\section{RÉFÉRENCES}

AFSSA. Avis de l'Agence française de sécurité sanitaire des aliments relatif à une demande d'évaluation des risques liés à la consommation courante d'huile de lin vierge, 31 mars 2009.

Agreste mai 2011. http://www.agreste.agriculture.gouv.fr,http://agreste.maapar.lbn.fr/ ReportFolders/ReportFolders.aspx.
Brunschwig P, Hurtaud C, Chilliard Y, Glasser F. L'apport de lin dans la ration des vaches laitières : effet sur la production, la composition du lait et des produits laitiers, les émissions de méthane et les performances de reproduction, INRA production. Animale $2010 ; 23: 307-18$.

Carluer-Lorssoruan F. Bleu Blanc Cœur : $40 \%$ de produits en plus d'ici 3 ans. Linéaires 01/04/2009 2009;15-6.

CETIOM. Lin graine d'hiver, septembre 2006. Brochure technique CETIOM, 2006, 4 p.

CETIOM. Lin graine de printemps, janvier. Brochure technique CETIOM, 2007, 4 p.

Collectif. Lin oléagineux. Semences et progrès $2010 ;(145): 81-3$.

Lessirard J. Amélioration de la qualité nutritionnelle des produits alimentaires. Rapport du Conseil général de l'agriculture, de I'alimentation et des espaces ruraux. CGAAER $\mathrm{n}^{\circ}$ 1824, 71 p, 2009.

Ministère de l'économie. Décret $\mathrm{n}^{\circ} 2008$ 184 du 26 février 2008 portant application du code de la consommation en ce qui concerne les graisses et huiles comestibles, 2009.

Ministère de l'économie. Arrêté du 4 décembre 2008 fixant les conditions d'utilisation de I'huile de lin pour un usage alimentaire. 